\title{
On-wall and interior separation in a two-fluid boundary layer
}

\author{
Sergei N. Timoshin $(\mathbb{D} \cdot$ Pallu Thapa
}

Received: 26 April 2019 / Accepted: 4 September 2019 / Published online: 23 September 2019

(C) The Author(s) 2019

\begin{abstract}
A two-fluid boundary layer is considered in the context of a high Reynolds number Poiseuille-Couette channel flow encountering an elongated shallow obstacle. The flow is laminar, steady and two-dimensional, with the boundary layer shown to have the pressure unknown in advance and a specified displacement (a condensed boundary layer). The focus is on the detail of the flow reversal triggered by the obstacle. The interface between the two fluids passes through the boundary layer which, in conjunction with the effects of gravity and distinct densities in the two fluids, leads to several possible topologies of the reversed flow, including a conventional on-wall separation, interior flow reversal above the interface, and several combinations of the two. The effect of upstream influence due to a transverse pressure variation under gravity is mentioned briefly.
\end{abstract}

Keywords Boundary-layer separation · High Reynolds numbers · Two-fluid flow

\section{Introduction}

This paper investigates the appearance of a local flow separation (reversed-flow vortex, region with closed streamlines-these terms are used interchangeably) in a two-fluid boundary layer. Such flows often arise in industrial, engineering and biophysical applications (e.g. on a rain-wetted aircraft wing, in mammals' airways, surface coating, industrial air blowers, etc.) and therefore command a great deal of theoretical interest, aiming to understand the interplay between various factors influencing the flow properties in a two-fluid configuration. Here the attention is restricted to flow separation; hence, the modelling is carried out for a laminar, steady and two-dimensional flow regime, paying attention to such factors as density stratification and gravitational forces but leaving aside questions of stability and laminar-turbulent transition.

The approach is based on a high Reynolds number asymptotic formulation known to give a detailed description of the physics of flow separation, first of all in the interaction between inviscid and viscous parts of the flow, e.g. [1-5]. A Poiseuille-Couette flow in an inclined channel is taken as a basis. Apart from immediate application to elongated geometries, the base flow can be viewed as a narrow-gap version of a two-fluid journal bearing [6] with related models found, for instance, in a study of a two-phase flow during liquid coating [7]. The main ideas to be discussed in the paper are easy to infer with reference to Fig. 1 which illustrates a channel with the upper wall moving at a

S. N. Timoshin $(\varangle) \cdot$ P. Thapa

Department of Mathematics, UCL, Gower St, London WC1E 6BT, UK

e-mail: s.timoshin@ucl.ac.uk 
constant speed and the lower wall at rest, resulting in a layered two-fluid current parallel to the channel walls. An obstacle on the lower wall creates transverse contractions (or expansions) of fluid particles which experience an additional acceleration (respectively-deceleration) most significant for the low-momentum current near the bottom wall. As is well known, this is where the flow tends to separate from the solid boundary. An immediate question to ask is whether the tendency for the flow to separate from a solid boundary persists in a two-fluid arrangement, especially if the interface passes close to the boundary and, moreover, the lower fluid has a greater density and therefore carries a higher momentum in relative terms. Intuitively, the answer will depend on whether the tendency for the lower, heavier fluid to cling to the wall under gravitational acceleration is sufficient to overcome the overall deceleration caused by the obstacle, leading to two plausible flow patterns with either lower fluid separating from the wall or the upper fluid reversing internally (for a quick illustration, the reader may want to look at Figs. 2a, 4d below). Beyond these predictable options, this paper attempts to investigate topological changes in the pattern of streamlines in the flow under variation of these competing trends.

In asymptotic analysis, the flow with a large Reynolds number, $\operatorname{Re} \gg 1$, is divided into a locally inviscid core covering the bulk of the channel and viscous sublayers near solid walls. This arrangement is typical for both Poiseuille and Couette flows or indeed for their combination; however, the way near-wall viscous layers communicate with each other is very sensitive to both the base-flow profile and the geometry of the obstacle. In a channel with a pure Poiseuille current, a most active interaction between the wall layers, also supported by the transverse pressure variation in the inviscid core, takes place on the streamwise length scale of $O\left(R e^{1 / 7}\right)$ identified in a sequence of papers [8-10]. As shown in [8], the flow regimes on longer and shorter scales are governed by somewhat simplified formulations closely related with the present investigation. The viscous layer near the moving upper wall in our situation proves passive. The viscous layer near the lower wall can then be described as a two-fluid version of a condensed boundary layer, condensed in the sense that the combined transverse displacement of both liquids in the near wall region vanishes. This is essentially due to the fact that the upper channel wall is rigid and has a non-zero speed, hence suppressing transverse movement of fluid particles. The concept of a boundary layer with a specified displacement and unknown in advance pressure (in effect-the opposite of the classical Prandtl's boundary layer where the pressure is specified and displacement is unknown) is well established. It appeared in early calculations of the wake behind a small wall-mounted obstacle in [11] and was then described in detail for the case of a compressible external flow [12] and an incompressible channel flow [8]. Condensed boundary layers are abundant in theoretical modelling of internal flows [3] but also have a wider applicability being, on the one hand, a natural short-scale limit of more general boundary layers with triple-deck type viscous/inviscid interaction and, on the other hand, a long-scale limit of the shear flow governed by full Navier-Stokes equations (see, e.g. [13], for the case of an incompressible external flow). Computations in [14] indicate that the concept of a condensed layer may become applicable at Reynolds numbers of order 100.

A two-fluid version of the viscous/inviscid (triple-deck) interaction theory closely related with this study is employed in [15] for the purposes of linear stability calculations, with a possibility of absolute instability in such flows discovered in [16]. Caporn [17] showed that the long-scale limit of the two-fluid triple-deck leads to a boundary layer with a specified pressure where, under significant deceleration, the flow was found typically to terminate with the formation of the Goldstein [18] singularity. In similarity with the case of a homogeneous fluid, the short-scale limit of the triple-deck is governed by a two-fluid condensed boundary layer. Computations in [17] are probably the first numerical solutions for a two-fluid condensed layer with separation which, in the absence of gravity or surface tension, is found to be of a conventional on-wall variety. At the same time, modelling the near-wall liquid layer using a lubrication approximation in [19] suggests that the flow reversal may take place in the interior of the upper fluid. Visualisation of coating flows in [20] reveals vortices in the near wall region or, alternatively, attached to the free surface of the layer of coating liquid. In [21], regions of mild recirculation are noted in the aerodynamic boundary layer on top of a liquid film with a nonlinearly distorted interface, see e.g. their Fig. 13. The flow considered is time-dependent and although the model used was also of an asymptotic nature, the details of the recirculation region are not very clear. Linear trends in the triple-deck model of a two-fluid flow were also re-examined recently in [22]. The flow type studied below in this paper is conceptually not far away from the two-fluid channel flow examined in [23] within the framework of full Navier-Stokes equations but with the interfacial surface tension included in the 


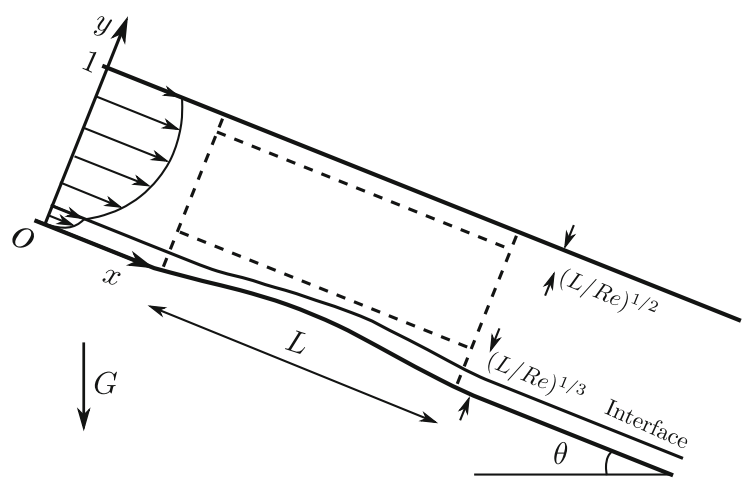

Fig. 1 Flow geometry, not to scale

model. In their results, an on-wall flow reversal is observed typically for the range of Reynolds numbers of several hundred.

There appear to be a few gaps in our understanding of two-fluid separated flows which this paper aims to bridge to some extent. The layout is as follows. In Sect. 2, the equations of motion are presented together with a brief description of the base two-fluid Poiseuille-Couette flow. The calculations are straightforward but are still needed to fix the notation in what follows. Some aspects of physics related to the density difference in the two fluids are emphasised to identify the relevant parameter ranges especially for a thin layer near the lower wall. The central formulation for the locally perturbed high Reynolds number flow past a wall roughness is given in Sect. 3. The relevant computational solutions are discussed in Sect. 4. An important property of a condensed boundary layer in a homogeneous fluid is the absence of upstream influence. This property holds in the two-fluid case as long as the hydrostatic pressure variation across the channel can be neglected, as will be the case for the most part in this paper, although a couple of examples of numerical solutions with upstream influence will be shown at the end of Sect. 4. Section 5 discusses a number of possible extensions of this work.

\section{A Poiseuille-Couette channel flow}

A schematic of the flow studied in this paper is shown in Fig. 1. The channel is infinitely long, with a fixed lower wall distorted by a local roughness and a straight upper wall moving with constant speed in the main flow direction as shown. The entire flow is laminar, time-independent and two-dimensional. In this section, the equations of motion are summarised and then, assuming that the wall roughness is absent, the velocity field in a parallel flow of two fluids is described. This provides a base state of the flow before it enters the region of wall irregularity.

The parameters used in non-dimensionalization are the speed of the moving wall, $U_{0}$, the channel width, $D$, and reference density $\rho_{0}$ and viscosity $\mu_{0}$ (later to be taken as those in the upper fluid). The velocity components along and normal to the channel are written as $U_{0} u^{ \pm}(x, y), U_{0} v^{ \pm}(x, y)$ respectively. Here $D x, D y$ denote the Cartesian coordinates along and normal to the channel and the sign in the superscript labels the fluid above (plus) and below (minus) the interface. The variable part of the pressure in the two fluids is written as $\rho_{0} U_{0}^{2} p^{ \pm}(x, y)$. The non-dimensional Navier-Stokes equations reflecting the two components of the momentum equation and the continuity are, in turn,

$$
\begin{aligned}
& \rho^{ \pm}\left(u^{ \pm} u_{x}^{ \pm}+v^{ \pm} u_{y}^{ \pm}\right)=-p_{x}^{ \pm}+\rho^{ \pm} G^{(x)}+\mu^{ \pm} R e^{-1}\left(u_{x x}^{ \pm}+u_{y y}^{ \pm}\right), \\
& \rho^{ \pm}\left(u^{ \pm} v_{x}^{ \pm}+v^{ \pm} v_{y}^{ \pm}\right)=-p_{y}^{ \pm}-\rho^{ \pm} G^{(y)}+\mu^{ \pm} R e^{-1}\left(v_{x x}^{ \pm}+v_{y y}^{ \pm}\right), \\
& u_{x}^{ \pm}+v_{y}^{ \pm}=0 .
\end{aligned}
$$

Here $R e=\rho_{0} U_{0} D / \mu_{0}$ is the Reynolds number, the dimensionless components of the body force due to gravity are $G^{(x)}=G \sin \theta, G^{(y)}=G \cos \theta$, with $G=g D U_{0}^{-2}, \theta$ is the inclination angle of the channel to the horizontal, 
$g$ is the magnitude of acceleration due to gravity and the non-dimensional densities and viscosities of the two fluids are denoted as $\rho^{ \pm}, \mu^{ \pm}$. The non-dimensional gravitational acceleration is $G=F r^{-2}$ in terms of the Froude number Fr.

A uni-directional flow is assumed in a channel of constant width so that $u^{ \pm}(x, y)=U^{ \pm}(y), v^{ \pm}(x, y)=0$ and $p^{ \pm}(x, y)=P^{ \pm}(x, y)$, say. It then follows that the pressure function can be written as

$P^{ \pm}=-\rho^{ \pm} G^{(y)}\left(y-y_{i}\right)+\pi_{0} x$,

with constant $\pi_{0}$, where $y=y_{i}$ marks the position of the interface. The pressure continuity at the interface has been applied with a constant of integration omitted. The constant $\pi_{0}$ appearing in this solution may be interpreted as a 'global' longitudinal pressure gradient due, for example, to an overall pressure difference between the ends of a long but finite channel. The pressure variation in the transverse direction has no effect on the base uni-directional flow but will become important when the wall roughness is introduced later on. If we denote

$\pi^{ \pm}=\operatorname{Re}\left(\rho^{ \pm} G^{(x)}-\pi_{0}\right) / \mu^{ \pm}$

then the longitudinal momentum equation reduces to a Poiseuille form in each fluid, $\mathrm{d}^{2} U^{ \pm}(y) / \mathrm{d} y^{2}=-\pi^{ \pm}$. In essence, the two fluids are driven by their own effective pressure gradients which are connected according to the relation,

$\mu^{-} \pi^{-}=\mu^{+} \pi^{+}+\operatorname{Re}\left(\rho^{-}-\rho^{+}\right) G^{(x)}$.

The solutions for the longitudinal velocity may be written as

$$
\begin{aligned}
& U^{+}(y)=D^{+}(y-1)+1-\frac{\pi^{+}}{2}\left(y^{2}-1\right), \\
& U^{-}(y)=D^{-} y-\frac{\pi^{-}}{2} y^{2},
\end{aligned}
$$

with constants $D^{ \pm}$determined by the interfacial conditions of the velocity and tangential stress continuity, i.e. $U^{+}=U^{-}$and $\mu^{+} \mathrm{d} U^{+} / \mathrm{d} y=\mu^{-} \mathrm{d} U^{-} / \mathrm{d} y$ at $y=y_{i}$. Complete expressions for $D^{ \pm}$are not very informative and are relegated to Appendix A.

The shape of the velocity profile $U^{+}(y)$ in the top fluid (7) is presented as a superposition of the linear Couette profile (with the coefficient $D^{+}$absorbing the effect of the layer of the bottom fluid) and the Poiseuille parabolic component. The specific flow type (e.g. pure Couette or predominantly Poiseuille or their various combinations) is controlled by the value of the constant $\pi_{0}$ in (5) which can be chosen to reduce or, by contrast, enhance the gravitational pull on the upper fluid by altering the value of $\pi^{+}$. In a similar manner, in the bottom fluid, the flow velocity $U^{-}(y)$ in (8) is a sum of the linear and quadratic terms in $y$. The important, if evident, fact is that even if the Poiseuille component is completely eliminated in the top layer (i.e. $\pi^{+}=0$ ), the density difference between the fluids creates an additional acceleration of the bottom fluid resulting in a parabolic velocity distribution near the lower channel wall $\left(\pi^{-} \neq 0\right.$ in (8)). This effect becomes more pronounced when the bottom layer is thin, as in the next sub-section.

\subsection{Thin film on a lower channel wall}

We are interested in the flow where most of the channel is filled with the upper fluid and the bottom fluid develops as a relatively thin film. At the same time the densities of the two fluids are sufficiently distinct for the film fluid to feel gravitational acceleration due to it's own weight in addition to the usual shearing effect of the top fluid. The 
thickness of the bottom layer is written as $y_{i}=\varepsilon a_{0}$ using $\varepsilon$ as an artificial scaling parameter, $0<\varepsilon \ll 1$, with the scaled film thickness $a_{0}=O(1)$. The effective pressure gradient in the bottom fluid is taken in the form

$\pi^{-}=\varepsilon^{-1} \bar{\pi}^{-}+O(1)$

with $\bar{\pi}^{-}=O(1)$. This ensures that both terms in (8) are of the same order of magnitude in the film where $y=O(\varepsilon)$ which in turn requires the pressure term $\operatorname{Re}\left(\rho^{-}-\rho^{+}\right) G^{(x)}=O\left(\varepsilon^{-1}\right)$ in (6). Hence the parameter range for this type of flow is narrowed down, and we shall quantify this further in the next section when the obstacle is introduced. In physical terms, the implication of (9) is that, despite small thickness of the film, viscous and gravitational forces remain in balance here.

The expansions presented in Appendix A show that the velocity field can now be divided into two regions. In the top fluid which now occupies the bulk of the channel, we have

$U^{+}(y)=U_{0}^{+}(y)+\varepsilon U_{1}^{+}(y)+O\left(\varepsilon^{2}\right)$,

where the first two coefficients are

$$
\begin{aligned}
& U_{0}^{+}(y)=y+\frac{\pi^{+}}{2}\left(y-y^{2}\right), \\
& U_{1}^{+}(y)=\left[a_{0}\left(1+\frac{\pi^{+}}{2}\right)\left(1-\frac{\mu^{+}}{\mu^{-}}\right)-\bar{\pi}^{-} \frac{a_{0}^{2}}{2}\right](y-1) .
\end{aligned}
$$

In the expansions above, it is assumed that the pressure gradient constant $\pi^{+}$is of $O(1)$.

Near the bottom channel wall, where we define $y=\varepsilon Y$ with the scaled coordinate $Y=O(1)$, the velocity field in the film fluid is

$U^{-}=\varepsilon\left[\lambda^{-} Y-\frac{\bar{\pi}^{-}}{2} Y^{2}\right]+O\left(\varepsilon^{2}\right)$

with the wall shear constant

$\lambda^{-}=\frac{\mu^{+}}{\mu^{-}}\left(1+\frac{\pi^{+}}{2}\right)+\bar{\pi}^{-} a_{0}$,

whereas for the adjacent part of the top fluid, we obtain

$U^{+}=\varepsilon\left[\lambda^{+}\left(Y-a_{0}\right)+u_{s 0}\right]+O\left(\varepsilon^{2}\right)$,

where the shear $\lambda^{+}$and the interfacial slip velocity $u_{s 0}$ are

$\lambda^{+}=1+\frac{\pi^{+}}{2}, \quad u_{s 0}=\frac{\mu^{+}}{\mu^{-}}\left(1+\frac{\pi^{+}}{2}\right) a_{0}+\frac{\bar{\pi}^{-}}{2} a_{0}^{2}$.

\section{Viscous-inviscid interaction}

We now assume that the Reynolds number in the flow is large and that the base Poiseuille-Couette flow from the previous section encounters a shallow obstacle located at the bottom wall as shown in Fig. 1. Within the framework of a high Reynolds number asymptotic theory, the aim is to establish a characteristic flow regime arising for a certain combination of the flow parameters: the Reynolds number $R e$, the scale of the film thickness $\varepsilon$, the gravitational 
acceleration $G$ and three geometric parameters, namely the wall slope $\theta$, the non-dimensional length scale of the obstacle denoted as $L$ and its height. There are clearly many interesting possibilities but our main concern is with the flow dynamics centred in the near-wall region. We therefore assume that the obstacle height is comparable with the film thickness $\varepsilon$ and then, from the balance between the inertia terms, pressure forces and viscosity in the longitudinal momentum equation (1), for the flow near the bottom wall where $y=O(\varepsilon)$ and $u^{ \pm}=O(\varepsilon)$, we have the following order of magnitude balances

$\varepsilon^{2} L^{-1} \sim p^{ \pm} L^{-1} \sim R e^{-1} \varepsilon^{-1}$

It follows that $\varepsilon=(L / R e)^{1 / 3}$ and $p^{ \pm}=O\left(\varepsilon^{2}\right)$. According to (4), an interface displacement of order $\varepsilon$ creates a gravitational pressure difference of order $\varepsilon^{2}$ if $G^{(y)}=O(\varepsilon)$, assuming in addition that the densities in the two fluids are comparable in magnitude. We therefore expect that the interfacial displacement will enter the near-wall dynamics provided that

$G \cos \theta=O(\varepsilon)$.

As follows from (6), the requirement (9) together with the condition $\pi^{+}=O(1)$ amounts to $\operatorname{Re} G^{(x)}=O\left(\varepsilon^{-1}\right)$ or

$G \sin \theta=O\left(\operatorname{Re}^{-1} \varepsilon^{-1}\right)$.

From these estimates,

$\tan \theta=O\left(\varepsilon^{-2} \operatorname{Re}^{-1}\right)=O\left(L^{-2 / 3} R e^{-1 / 3}\right)$,

where the last expression contains the length scale of the wall obstacle.

Based on the estimates above, several typical flow regimes arise for various choices of the obstacle length. For a very short obstacle, for example, with $L=O\left(R e^{-1 / 2}\right)$, the thickness of the near wall viscous layer is of the same order as its typical length, $\varepsilon=(L / R e)^{1 / 3}=O\left(R e^{-1 / 2}\right)$ and it is easy to verify that the local flow is then governed by the complete system of the Navier-Stokes equations. The angle of inclination is then $\theta=O(1)$ according to (20). In the opposite limit of a long obstacle, with $L=O(R e)$ and the slope of the channel $\theta=O\left(R e^{-1}\right)$, we have $\varepsilon=O(1)$ which means that the flow across the entire channel is governed by the boundary-layer equations with the interface located somewhere in the middle of the channel. Our main interest is in the intermediate length scales. To make presentation somewhat more palatable (with straightforward generalisations), we assume in addition that the obstacle is long relative to the channel width, i.e.

$1 \ll L \ll R e$.

It should be noted that, unlike the asymmetrically distorted Poiseuille flow in [10], the Couette component in our flow prevents the pressure variation across the channel from entering the leading-order balances as explained in Appendix B.

The links between the flow parameters in (17)-(19) lead to the scaling,

$G=\varepsilon G_{0}, \quad \theta=\varepsilon^{-2} R^{-1} \theta_{0}$,

where $G_{0}, \theta_{0}$ are of $O(1)$. The gravitational acceleration components expand as $G^{(x)}=\varepsilon^{-1} R e^{-1} G_{0} \theta_{0}+\cdots$, $G^{(y)}=G_{0}+\cdots$.

For the flow in the near-wall region in Fig. 1, we define local variables, $x=L X, y=\varepsilon Y$, with $(X, Y)=O(1)$ and recall that $\varepsilon=(L / R e)^{1 / 3}$. The flow functions at leading order are of the form:

$$
u^{ \pm}=\varepsilon u_{1}^{ \pm}(X, Y)+\cdots, \quad v^{ \pm}=\varepsilon^{2} L^{-1} v_{1}^{ \pm}(X, Y)+\cdots,
$$




$$
p^{ \pm}=\varepsilon^{2}\left[-\rho^{ \pm}\left(Y-a_{0}\right)+\rho^{+} G_{0} \theta_{0} X+p_{1}^{ \pm}(X, Y)\right]+\cdots .
$$

Here the extra terms appearing next to $p_{1}^{ \pm}$are introduced to simplify the equations below, and we ignore an overall additive constant in the pressure.

Substitution into (1)-(3) leads to the boundary-layer system of equations,

$$
\begin{aligned}
& \rho^{ \pm}\left(u_{1}^{ \pm} u_{1 X}^{ \pm}+v_{1}^{ \pm} u_{1 Y}^{ \pm}\right)=-p_{1 X}^{ \pm}+\left(\rho^{ \pm}-\rho^{+}\right) G_{0} \theta_{0}+\mu^{ \pm} u_{1 Y Y}^{ \pm}, \\
& u_{1 X}^{ \pm}+v_{1 Y}^{ \pm}=0,
\end{aligned}
$$

with the transverse momentum equation showing that the additional pressure terms do not vary across the viscous layer, $p_{1}^{ \pm}=p_{1}^{ \pm}(X)$, since the gravity-induced hydrostatic component is already included in (21).

Let the shape of the wall obstacle and the position of the interface be given by the relations $y=\varepsilon h_{1}(X)$ and $y=\varepsilon f_{1}(X)$, respectively. The no-slip conditions for the lower fluid on the solid boundary are

$u_{1}^{-}=v_{1}^{-}=0$ at $Y=h_{1}(X)$.

At the interface, we require continuity of the velocity and stress components together with the kinematics condition which for the leading-order terms reduce to

$u_{1}^{+}=u_{1}^{-}, \quad v_{1}^{ \pm}=u_{1}^{ \pm} f_{1}^{\prime}(X), \quad \mu^{+} u_{1 Y}^{+}=\mu^{-} u_{1 Y}^{-}$,

$p_{1}^{+}-p_{1}^{-}=G_{0}\left(\rho^{+}-\rho^{-}\right)\left(f_{1}-a_{0}\right)$ at $Y=f_{1}(X)$,

where the prime denotes the $X$-derivative.

The undisturbed flow field upstream of the obstacle is given by the relations,

$$
\begin{aligned}
& u_{1}^{+} \rightarrow \lambda^{+}\left(Y-a_{0}\right)+u_{s 0}, \quad v_{1}^{+} \rightarrow 0 \quad \text { as } X \rightarrow-\infty, Y>a_{0}, \\
& u_{1}^{-} \rightarrow \lambda^{-} Y-\frac{\bar{\pi}^{-}}{2} Y^{2}, \quad v_{1}^{-} \rightarrow 0 \quad \text { as } X \rightarrow-\infty, 0 \leq Y<a_{0},
\end{aligned}
$$

where $\bar{\pi}^{-}=\left(\rho^{-}-\rho^{+}\right) G_{0} \theta_{0} / \mu^{-}$, the shear coefficients $\lambda^{ \pm}$and the velocity at the interface are given in (14), (16) and the interface in the incoming stream is located at $Y=f_{1}(-\infty)=a_{0}$.

Far from the interface, the upper fluid exhibits a typical viscous sublayer behaviour,

$u_{1}^{+}=\lambda^{+}\left(Y-a_{0}\right)+u_{s 0}+A_{1}(X)+o(1)$ as $Y \rightarrow \infty$,

the displacement function $A_{1}(X)$ being part of the solution.

The problem formulation is closed by connecting the pressure function in the upper fluid $p_{1}^{+}$with the displacement function $A_{1}(X)$. The analysis presented in Appendix B shows that in the present case the displacement effect vanishes,

$A_{1}(X)=0$

with the viscous sublayer in the so-called condensed-flow regime of viscous/inviscid interaction. We note that the zero-displacement requirement applies also to certain types of a perturbed Poiseuille flow, except in our case the effect is exaggerated by the fact that the viscous sublayers on the opposite walls do not interact with each other directly as shown in Appendix B, cf. [8,9]. 


\section{Numerical solutions}

\subsection{Scaled formulation}

The boundary-value problem (22)-(28) is now re-written in the form suitable for a numerical treatment. First, we set $\rho^{+}=\mu^{+}=1$ thereby taking the density and viscosity of the upper fluid as reference quantities and denote $\rho^{-}, \mu^{-}$as $\rho, \mu$, respectively. The base-flow shear $\lambda^{+}$can be eliminated by the following change of variables where, emphatically, the notation in the transformed variables has a different meaning to that in the original Navier-Stokes equations at the beginning of the paper. For an arbitrary $\alpha>0$, let

$$
\begin{aligned}
& X=\alpha^{3} \lambda^{+} x, Y=\alpha y, u_{1}^{ \pm}=\alpha \lambda^{+} u^{ \pm}, v_{1}^{ \pm}=\alpha^{-1} v^{ \pm}, p_{1}^{ \pm}=\alpha^{2}\left(\lambda^{+}\right)^{2} p^{ \pm}, \\
& h_{1}=\alpha h(x), f_{1}=\alpha f(x), a_{0}=\alpha a, u_{s 0}=\alpha u_{s}, \\
& G_{0}\left(\rho^{-}-\rho^{+}\right)=\alpha\left(\lambda^{+}\right)^{2} g, \quad\left(\rho^{-}-\rho^{+}\right) G_{0} \theta_{0}=\alpha^{-1} \lambda^{+} K .
\end{aligned}
$$

The free parameter $\alpha$ may be used to accommodate specific modelling aims and eliminate a certain parameter from the problem formulation. For our purposes, it is convenient to assume that the wall obstacle has a certain 'length', $X=L_{0}$ say, then the first of the above transformations with $\alpha^{3} \lambda^{+}=L_{0}$ reduces the wall geometry to a canonised shape with an obstacle of unit length and varying height.

The formulation for the fluid above the interface, for $y>f(x)$, includes the boundary-layer equations,

$u^{+} u_{x}^{+}+v^{+} u_{y}^{+}=-p_{x}^{+}+u_{y y}^{+}, u_{x}^{+}+v_{y}^{+}=0, \quad p^{+}=p^{+}(x)$,

with the zero-displacement condition at the outer edge,

$u^{+}=y-a+u_{s}+o(1) \quad$ as $y \rightarrow \infty$,

and the upstream conditions,

$u^{+}=y-a+u_{s}+o(1), \quad v^{+} \rightarrow 0, p^{+} \rightarrow 0 \quad$ as $x \rightarrow-\infty$.

The equations in the lower fluid are

$\rho\left(u^{-} u_{x}^{-}+v^{-} u_{y}^{-}\right)=-p_{x}^{-}+K+\mu u_{y y}^{-}, u_{x}^{-}+v_{y}^{-}=0, \quad p^{-}=p^{-}(x)$,

to be solved in the strip $h(x)<y<f(x)$ subject to the no-slip wall conditions,

$u^{-}=v^{-}=0 \quad$ at $y=h(x)$,

and the conditions in the incoming stream,

$u^{-}=\lambda y-(2 \mu)^{-1} K y^{2}+o(1), v^{-} \rightarrow 0, p^{-} \rightarrow 0$ as $x \rightarrow-\infty$,

where

$\lambda=(1+K a) / \mu, \quad u_{s}=a / \mu+K a^{2} /(2 \mu)$.

It is assumed that the obstacle is localised and that the interface is flat far upstream so that $h(x) \rightarrow 0, f(x) \rightarrow a$ as $x \rightarrow-\infty$. The final set of the boundary conditions apply at the interface and include the continuity of the velocity field and the kinematic condition,

$u^{+}=u^{-}, \quad v^{+}=v^{-}, \quad v^{ \pm}=f^{\prime}(x) u^{ \pm} \quad$ at $y=f(x)$, 
together with the continuity of the stress components,

$u_{y}^{+}=\mu u_{y}^{-}, \quad p^{+}-p^{-}=-g(f-a) \quad$ at $y=f(x)$.

The boundary-value problem (30)-(37) is central to this paper. To recap, the flow is in the viscous sublayer where the impact of the wall obstacle (with the shape given by $y=h(x)$ ) is most significant. The incoming stream consists of a liquid film adjacent to the wall and a constant-shear current of a different fluid above, with the interface between the two fluids located initially at $y=a$ and then acquiring a shape given by $y=f(x)$ under the influence of the obstacle. The fluid below the interface is driven in part by the shear in the upper fluid and in part by the longitudinal gravitational component $K$. The pressure terms $p^{ \pm}$in the two fluids represent corrections to the hydrostatic pressure distribution and the overall external pressure in the channel. The last condition in (37) stems from the overall pressure continuity at the interface. Apart from the obstacle shape, the formulation contains five parameters: the incoming film thickness $a$, the relative viscosity $\mu$ and density $\rho$ of the film fluid, and two components of the gravitational acceleration, along the wall, $K$, and in the transverse direction, $g$. Depending on the channel orientation and the densities of the two fluids the quantities $K$ and $g$ can be positive or negative; however, in this paper, we assume a stable density stratification with the more dense fluid in the film and, in addition, the channel tilted downwards as in Fig. 1 to ensure that both $K$ and $g$ are positive. The implication, according to (29), is that in the undisturbed state the current in both fluids is from left to right (relative to the $x$-axis). The viscous sublayer problem is solved numerically using a second-order finite-difference method described in Appendix C.

\subsection{Flow without gravity}

In the illustrations below, the wall obstacle is taken in a Gaussian shape,

$h=h_{0} \exp \left(-x^{2}\right)$.

When the gravitational influences are absent, we have $g=K=0$ and the incoming flow has a two-fluid Couette velocity profile. This is a fairly benign situation exemplified in Fig. 2 where (as in subsequent diagrams) the position of the interface is indicated with dashes. In this case, $\rho=1.5, \mu=2.25$ although qualitatively similar flow patterns were observed for other combinations of the flow parameters. Note that the streamfunction in all illustrations below is not distributed uniformly as there is a significant difference in the velocity of reversed and forward flow. For this reason, the value of the streamfunction will not be shown by the individual curves to avoid clutter.

This flow regime was considered in [17] where an example of a separated flow similar to that in Fig. 2c albeit for a considerably thicker film is given. In all our computations with varying viscosity and density in the film and changing incoming film thickness, the flow separation takes place on the wall as in the case of a homogeneous fluid.

Reduction in the film thickness leads to an interesting limit $a \rightarrow 0$ where, despite a very small flux in the film, the entire separation bubble appears to be filled with the film fluid. Indeed, Fig. $2 \mathrm{~b}$ and d drawn for $a=0.05$ show that, apart from the small areas near separation and reattachment points, the interface is virtually indistinguishable from the separated streamline.

\subsection{Effect of channel slope: internal flow reversal}

Assuming as before that the pressure difference across the interface is absent (i.e. $g=0$ in (37)), the channel slope is now included by taking non-zero values of $K$. Fig. 3 shows the effect of increasing $K$ in the flow with a thin film $(a=0.5)$ of a relatively dense and viscous fluid with $\rho=4, \mu=8$. The wall has an indentation of depth $h_{0}=-3.5$.

As the momentum of fluid particles in the film increases with the increasing slope of the channel, the tendency in the film fluid to separate diminishes (Fig. 3b). As shown in Fig. 3c, the reversed-flow vortex may even be completely 


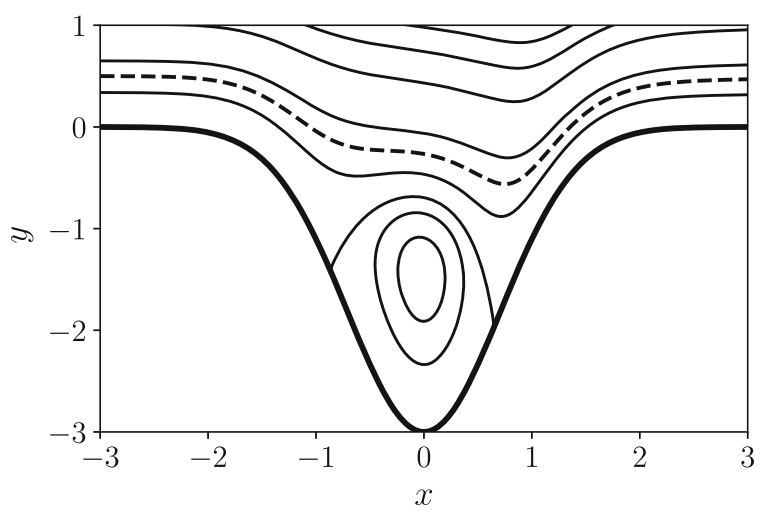

(a) $h_{0}=-3, a=0.5$

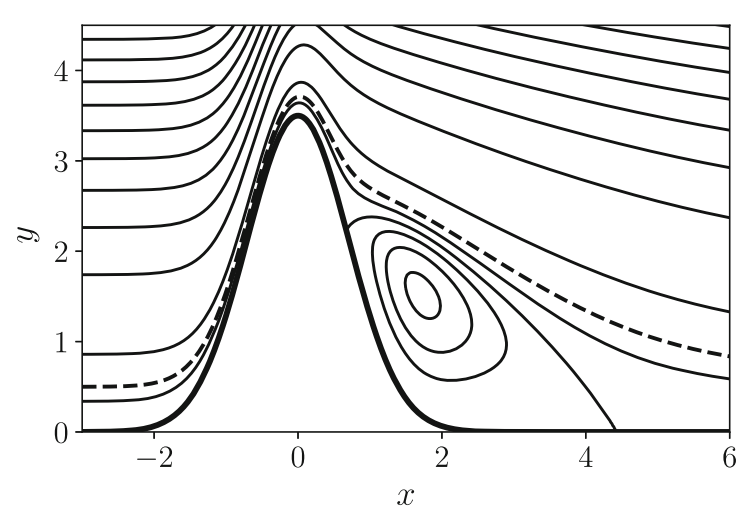

(c) $h_{0}=3.5, a=0.5$

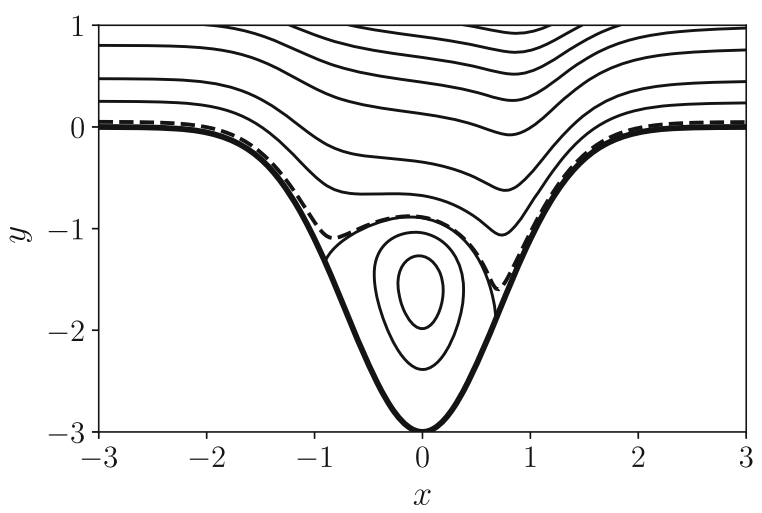

(b) $h_{0}=-3, a=0.05$

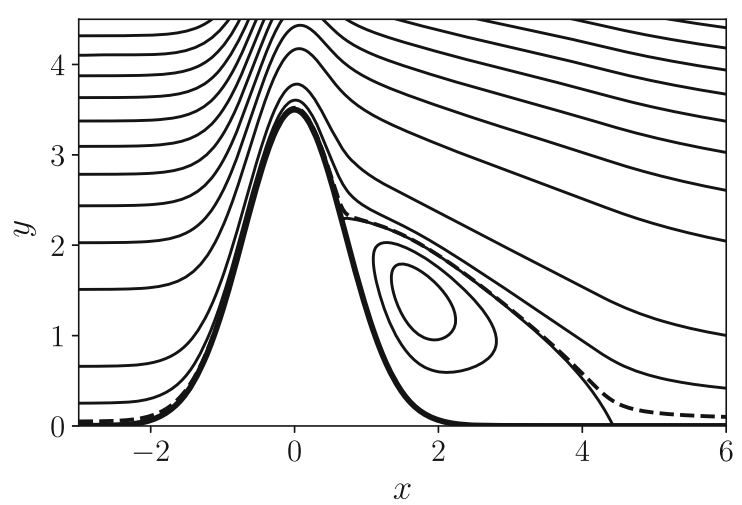

(d) $h_{0}=3.5, a=0.05$

Fig. 2 Flow separation in the absence of gravitational forces. $\mathbf{a}$ and $\mathbf{b}$ are for the flow past an indentation, $\mathbf{c}, \mathbf{d}$ are for the case of a hump. The interface between two fluids is shown with dashes

eliminated. However, as the channel slope increases further and the film becomes thinner in the indentation area, the flow field above the interface experiences a stronger transverse expansion leading to a re-appearance of the reversed-flow bubble, this time above the interface (Fig. 3d). In order to gain a better understanding of the flow topology at the origin of the interior separation, in the next subsection, we address a limiting case of a thin liquid film flowing past the obstacle under a strong gravitational acceleration.

\subsection{Limit of a thin heavy film}

Let $K \rightarrow \infty, g=O(1)$, and simultaneously $a \rightarrow 0$ with the film thickness scaling set as $a=K^{-1 / 2} \bar{a}$, with $\bar{a}=O(1)$. In the film flow, we have $y=h(x)+K^{-1 / 2} \bar{Y}, \bar{Y}=O(1)$, and $u^{-}=\bar{U}(x, \bar{Y})+\ldots, v^{-}=$ $u^{-} h^{\prime}(x)+K^{-1 / 2} \bar{V}(x, \bar{Y})+\cdots$, where the leading-order velocity components are found to be

$\bar{U}=\left(2 \bar{a} \bar{Y}-\bar{Y}^{2}\right) /(2 \mu), \bar{V}=0$.

This limiting flow corresponds to a liquid film with a free surface passively following the wall shape. The film thickness remains constant at leading order so that $f(x)=h(x)+K^{-1 / 2} a+\cdots$, essentially because longitudinal changes in the pressure $p^{-}$are of $O(1)$ and do not enter the leading-order momentum balance. The main effect of the flow is in creating an effective slip velocity for the outer fluid, $\bar{U}=\bar{a}^{2} /(2 \mu)$ at $\bar{Y}=\bar{a}$. The problem formulation 


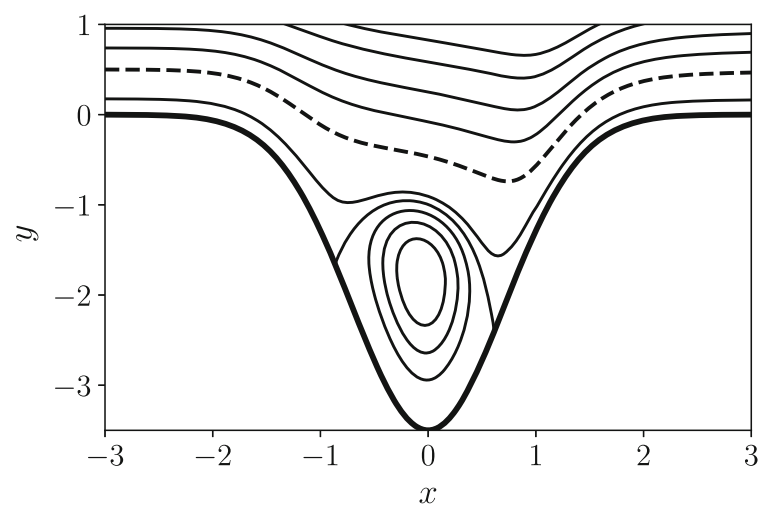

(a) $K=0.1$

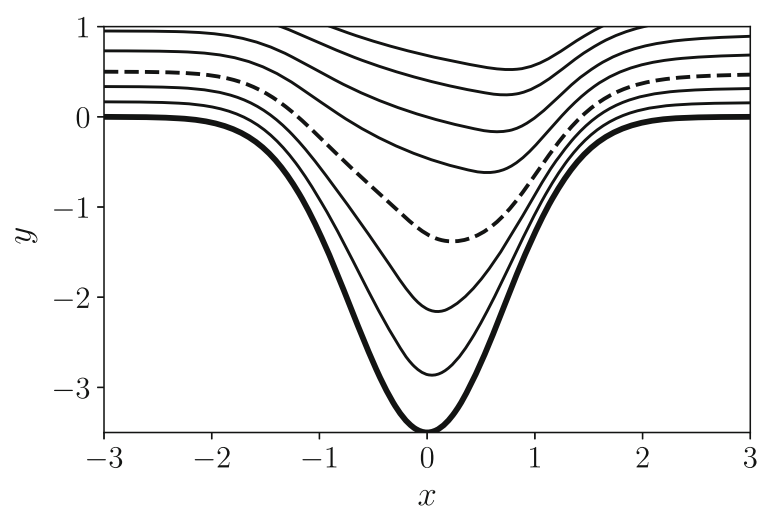

(c) $K=0.35$

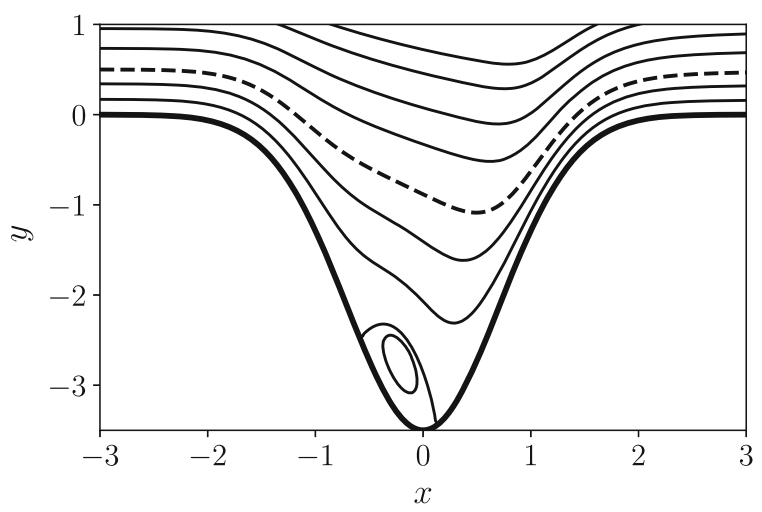

(b) $K=0.25$

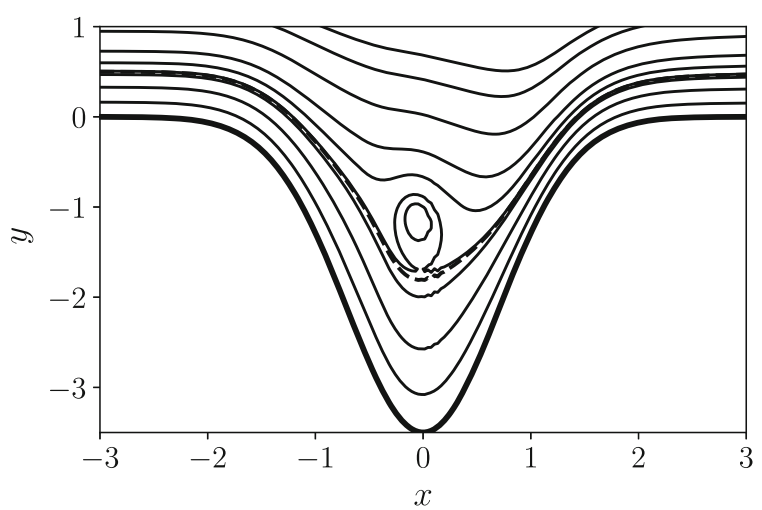

(d) $K=0.48$

Fig. 3 Degeneration of the on-wall separation under the influence of the channel slope followed by the appearance of an internal separation above the interface. The interface is shown with dashes

for to upper fluid now reduces to the single-fluid condensed boundary layer governed by Eq. (30) with the outer-edge boundary condition $u^{+}=y+\bar{u}_{s}+o(1)$ as $y \rightarrow \infty$, the incoming current given as a Couette flow on a sliding wall, $u^{+} \rightarrow y+\bar{u}_{s} x \rightarrow-\infty$, and the wall conditions corresponding to the given slip,

$u^{+}=\bar{u}_{s}, v^{+}=\bar{u}_{s} h^{\prime}(x) \quad$ at $y=h(x)$.

The magnitude of the wall slip, from matching with the film flow, is given by

$\bar{u}_{s}=\bar{a}^{2} /(2 \mu)$.

Examples of numerical solutions are shown in Fig. $4 \mathrm{a}-\mathrm{c}$ for the obstacle shape $h(x)=h_{0} \exp \left(-x^{2}\right), \bar{u}_{s}=0.15$ and several values of $h_{0}$. Note that the solution does not experience the moving-wall singularity of the type described in [24] at the first appearance of flow reversal since the pressure function $p^{+}$is not specified in advance. However, as the depth of the indentation increases, the shape of the streamlines at the incipient vortex develops a cusp (Fig. 4b), which subsequently transforms into a vortex with a saddle point on the dividing streamline.

To make a further comparison and estimate the validity of the thin-film approximation, Fig. 4d illustrates a full twofluid computation for the case $K=25, \mu=2, \rho=4, a=0.15492, h_{0}=-3.5$. In this case, $\bar{a}=a K^{1 / 2}=0.7746$, $\bar{u}_{s}=0.15$ placing the solutions in Fig. $4 \mathrm{c}$ and d roughly in the same parameter range. Although there is some quantitative disagreement between the two diagrams, the location of the vortex appears to be captured correctly. 


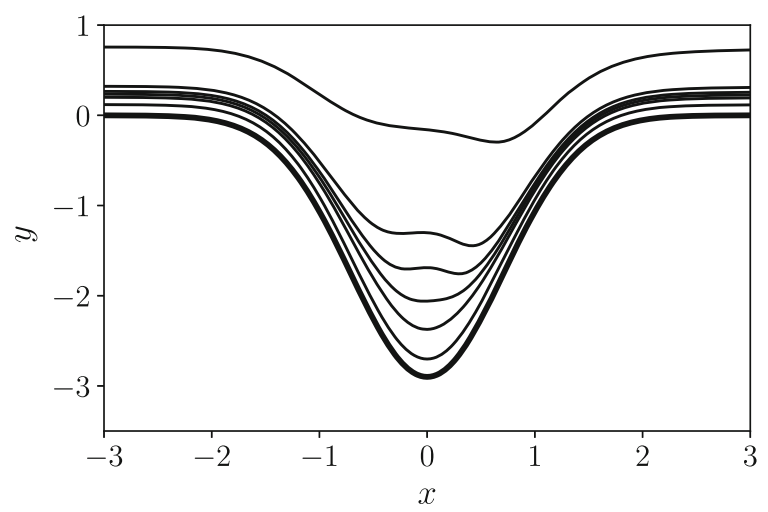

(a) $h_{0}=-2.9$

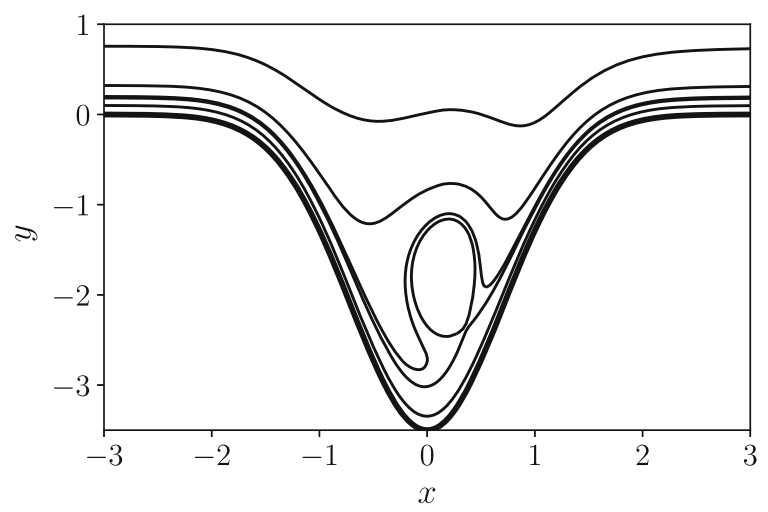

(c) $h_{0}=-3.5$

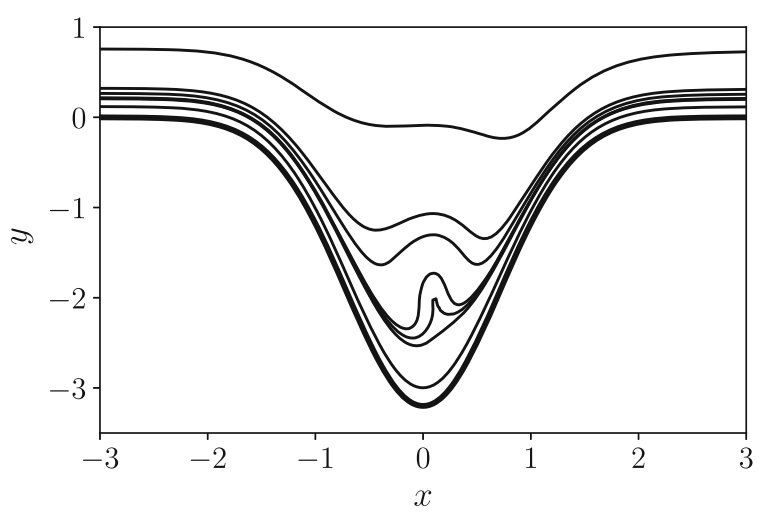

(b) $h_{0}=-3.2$

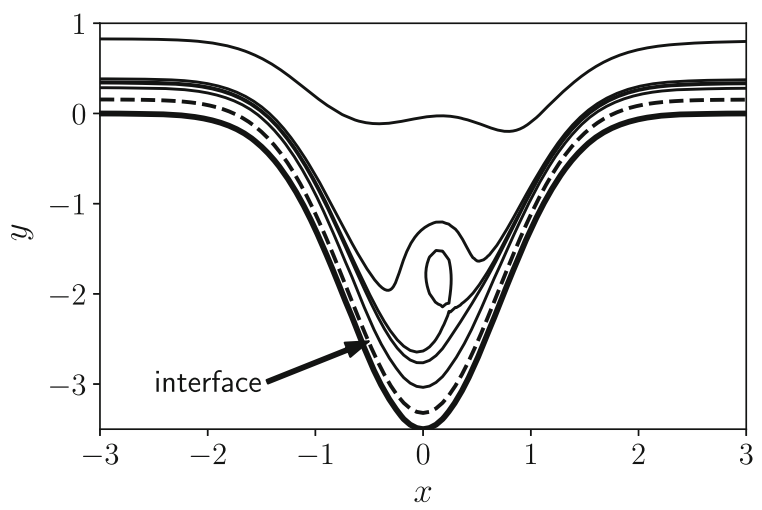

(d) Two-fluid flow, $h_{0}=-3.5$

Fig. 4 The wall shape is shown with a solid curve. a-c solutions of the moving-wall limiting problem; d two-fluid formulation, the dashes show the interface

\subsection{Topology of the incipient interior separation}

The patterns of streamlines at the first appearance of a recirculation region in the interior can be deduced qualitatively from a local form of the streamfunction, here denoted as $\psi$ for simplicity. Assuming rather generally that the flow field is sufficiently smooth, suppose the problem formulation contains a parameter, $A$ say, such that $A=0$ corresponds to a transition between the flow pattern with and without recirculation region. At small values of $A$ the streamfunction $\psi=\psi(x, y ; A)$ expands as $\psi=\Psi_{0}(x, y)+A \Psi_{1}(x, y)+O\left(A^{2}\right)$, where the leading-order term $\Psi_{0}$ contains a stationary point located, without loss of generality, at the origin at $x=y=0$. Near the origin, a local expansion can be written in the form

$\Psi_{0}=a_{20} x^{2}+a_{21} x y+a_{22} y^{2}+\sum_{i=0}^{3} a_{3 i} x^{3-i} y^{i}+\ldots$,

with constant coefficients $a_{i j}$, keeping only the quadratic and cubic terms. This form is consistent with the boundarylayer equations which are third-order in the transverse coordinate and, in addition, contain the pressure function unspecified in this analysis. Depending on the coefficients of the quadratic form, the stationary point is a saddle or a centre, neither of which is a suitable candidate for our task. The third option is a degenerate form in which, after a suitable rotation of axes, we can take $a_{21}=a_{22}=0$ hence giving the quadratic term in the streamfunction 


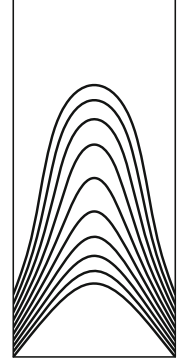

(a) Interior of the flow, fold bifurcation
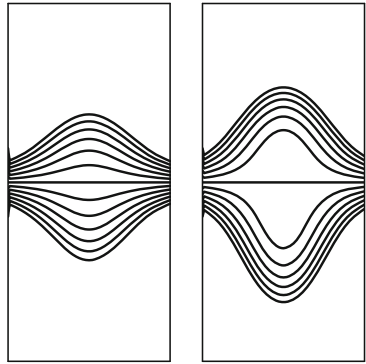

(c) Asymmetric interfacial vortex
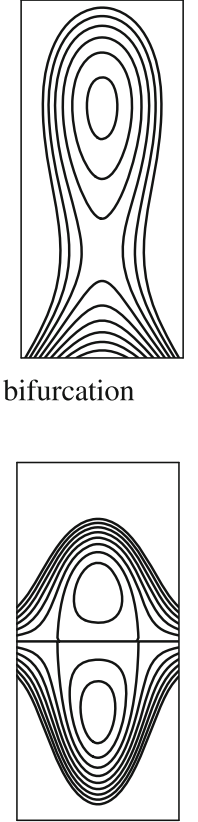
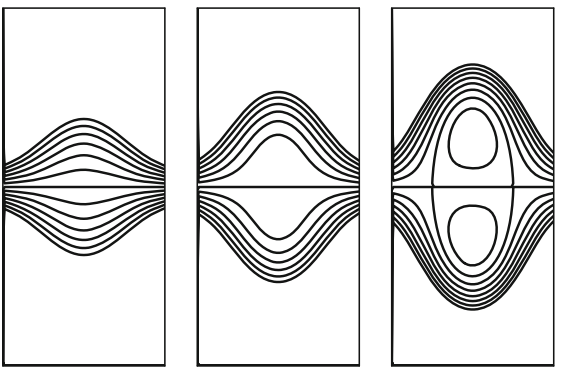

(b) Symmetric interfacial vortex
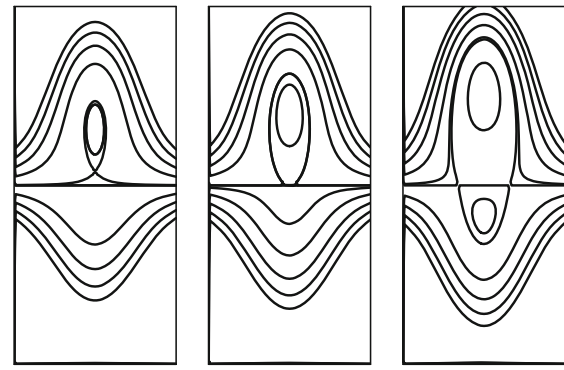

(d) Vortex migrating through interface

Fig. 5 a-c show local streamlines with an incipient recirculation region. In case d, a saddle-centre region with closed streamlines transforms into a twin saddle-centre structure of a double-vortex

expansion as a single term $a_{20} x^{2}$. In fluid-dynamical terms this corresponds to a set of streamlines parallel to the $y$-axis (however, this is only after the assumed rotation of axes). The shape of the streamlines is therefore determined by the cubic terms in (38). Order-of-magnitude considerations show that the first significant term locally is $a_{23} y^{3}$ thus leading to the expansion

$\Psi_{0}=a_{20} x^{2}+a_{23} y^{3}+\cdots$,

with the expected cusp shape of the streamline $\Psi_{0}=0$ of the form $y \sim x^{2 / 3}$ and the cusp orientation dependent on the signs of the constant coefficients.

The cusp formation is not structurally stable to variations in the control parameter $A$. The corresponding correction term in the streamfunction can be written as $\Psi_{1}=b_{10} x+b_{11} y+\cdots$. Here, we omitted an insignificant additive constant and denote the constant coefficients as $b_{i j}$. Note that, from the balance, $y=O\left(x^{2 / 3}\right)$, the term $b_{10} x$ can be neglected locally. In summary, a locally perturbed streamfunction near the stationary point takes the form,

$\psi=a_{20} x^{2}+a_{23} y^{3}+\cdots+A\left(b_{11} y+\cdots\right)+O\left(A^{2}\right)$.

As an example, Fig. 5a illustrates, from left to right, three cases, $\psi=y^{3}+x^{2}+y / 2, \psi=y^{3}+x^{2}$, and $\psi=y^{3}+x^{2}-y / 2$, with the middle pane demonstrating the stationary point at the tip of the cusp giving rise to an interior separation region via a fold (more exactly—saddle-centre) bifuraction; see e.g. [25] and references therein for a related discussion of certain solutions of the Navier-Stokes equations.

The sensitivity of the cusp in the streamline pattern is evident in computations in Fig. 4 where, with variations in the obstacle depth, the local field switches readily from a regular pattern to a recirculating region with a centre-saddle pair.

A similar analysis can be carried out for the incipient recirculation in flows restricted by various symmetries and allows even broader extensions. Of particular interest is the behaviour of a recirculation region surrounding the interface (relevant computational results will be discussed in the next sub-section). Assuming that the $x$-axis is a locally flat interfacial streamline $\psi=0$, the analysis above needs to be altered with the term $a_{20} x^{2}$ removed and the focus placed on the structure of the cubic terms. Assuming, to begin with, a left-right and up-down 
symmetries in the local flow field, the streamfunction expansion at the stationary point placed at the origin reduces to $\Psi_{0}=a_{33} y^{3}+a_{31} x^{2} y+\cdots$. With extra perturbations due to variations in global parameters (modelled locally by a single small parameter $A$ as before), the streamfunction takes the form:

$\psi=a_{33} y^{3}+a_{31} x^{2} y+\cdots+A\left(b_{11} y+\cdots\right)+b_{22} y^{2}+\cdots+O\left(A^{2}\right)$.

As an additional perturbation, here we introduced the term $b_{22} y^{2}$ responsible for breaking the flow symmetry relative to the $x$-axis. For consistency, the value of $b_{22}$ has to be small. If the $x$-axis separates two different fluids, an expression similar to (39) can be derived below the interface using the velocity and stress continuity conditions. We can also take into account a possible difference in the longitudinal pressure gradient at the interface. Hence (39) will now be valid for $y>0$, we whereas at $y<0$, we can write

$\psi=a_{33}^{-} y^{3}+a_{31} x^{2} y+\cdots+A\left(b_{11} y+\cdots\right)+\mu^{-1} b_{22} y^{2}+\cdots+O\left(A^{2}\right)$,

where $a_{33}^{-}$is a constant related to the pressure gradient and the relative viscosity coefficient below the interface is $\mu$.

In Fig. 5b, the streamfunction $\psi=y^{3}+x^{2} y+b y$ is shown for $b=0.25,0$ and -0.25 (left-to-right, respectively). This illustrates a homogeneous fluid flow with a double-vortex appearing on the zero streamline by virtue of a twin saddle-centre bifurcation (see, e.g. [26]). A similar sequence can also be expected in a two-fluid flow illustrated in Fig. $5 \mathrm{c}$ where $\psi=y^{3}+x^{2} y+$ by for $y>0, \psi=y^{3} / 2+x^{2} y+b y$ for $y<0$ with $b=0.25,0,-0.25$. Including the symmetry breaking, Fig. $5 \mathrm{~d}$ shows how an interior vortex may be transformed by the interface into a vortex pair. In this illustration, $\psi=y^{3}+x^{2} y+b y-y^{2} / 2$ for $y>0, \psi=y^{3} / 2+x^{2} y+b y-y^{2} / 4$ for $y<0$, with the value of $b$ taken as $0.05,0$ and -0.1 in the diagrams from left to right, respectively. The case of a recirculation vortex appearing on the solid wall with the conditions $\psi=\partial \psi / \partial y=0$ at $y=0$ can be examined in a similar fashion.

The patterns illustrated in this sub-section are not exhaustive. The axes rotation, for instance, does not change the local topology but gives a different perspective from the point of view of the overall flow. As an example, rotation of the cusp in Fig. 5a anticlockwise generates a pattern with reversed flow (in a predominant left-to-right current) but not necessarily with closed streamlines. It should be noted that the boundary-layer equations are not rotation-invariant; however, as long as the $y$-dependence in the streamfunction is limited to cubic terms in $y$, regular local expansions remain valid. Alternative bifurcations are obtained by choosing suitable polynomial forms in the coordinate expansions. Whether any such patterns arise in the solution of any given boundary-value problem is a different matter altogether.

\subsection{Vortex on the interface}

The local analysis in the previous subsection indicates that a reversed-flow region may have a two-cell structure surrounding the interface as in Fig. 5c, d. We now return to the two-fluid boundary-layer problem and examine flow regimes where similar structures appear in numerical solutions. In Fig. 6, the channel is inclined at a fixed angle (here $K=0.75, \rho=1.5, \mu=2.25, h_{0}=-3.25$ ) and the film thickness $a$ varies. For a relatively thin film with $a=0.05$ in Fig. 6a, the larger vortex in the upper fluid is joined at the interface with a slender vortex in the film. The enlarged section of the flow field in Fig. $6 \mathrm{~b}$ shows the streamlines near the upstream saddle point on the interface-notice how the flow reversal in the upper fluid (i.e. negative values of the longitudinal velocity) appears quite far ahead of the flow reversal in the film, even though both events are related in terms of the streamline topology.

As the film thickness increases to $a=0.3$, the vortex in the film becomes smaller and eventually vanishes, leaving a single reversed flow zone in the upper fluid (Fig. 6c and d). This is essentially the sequence of events in the local model in Fig. 5d only in reverse order. 


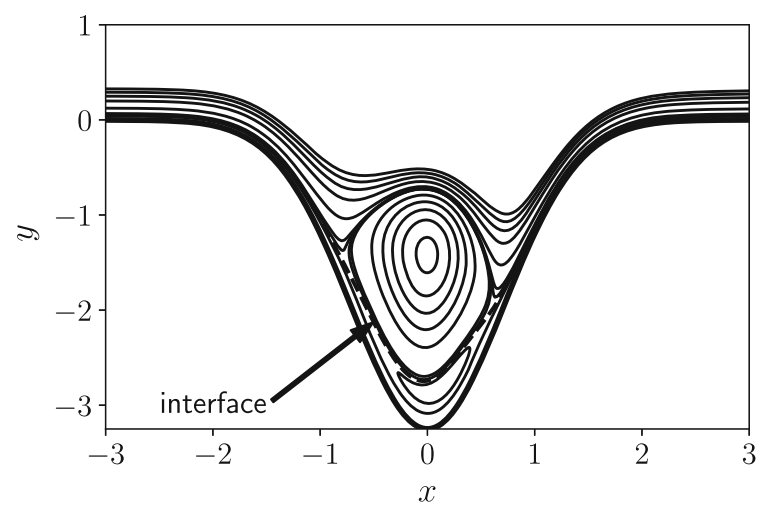

(a) $a=0.05$

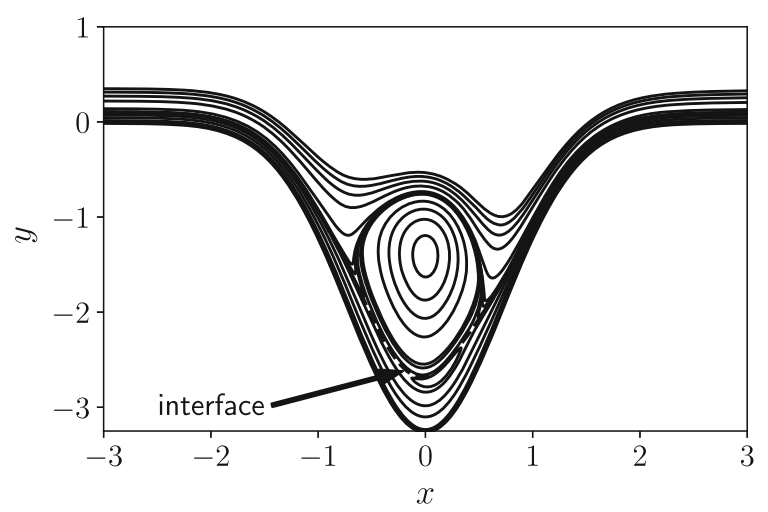

(c) $a=0.1$

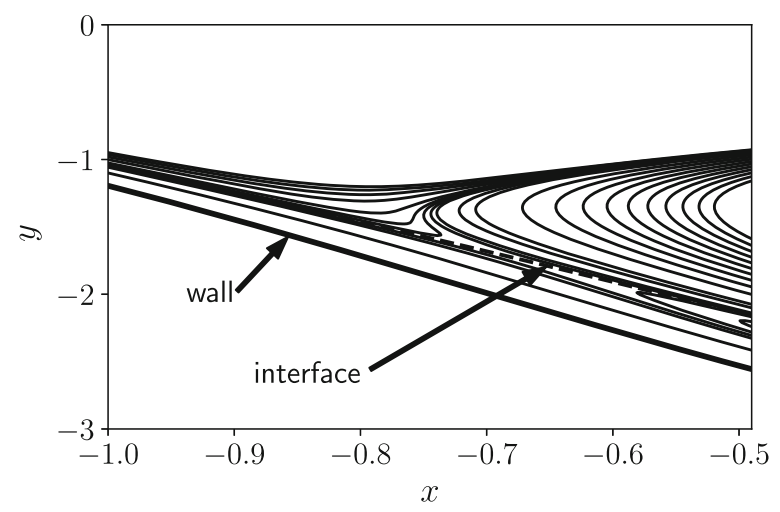

(b) $a=0.05$

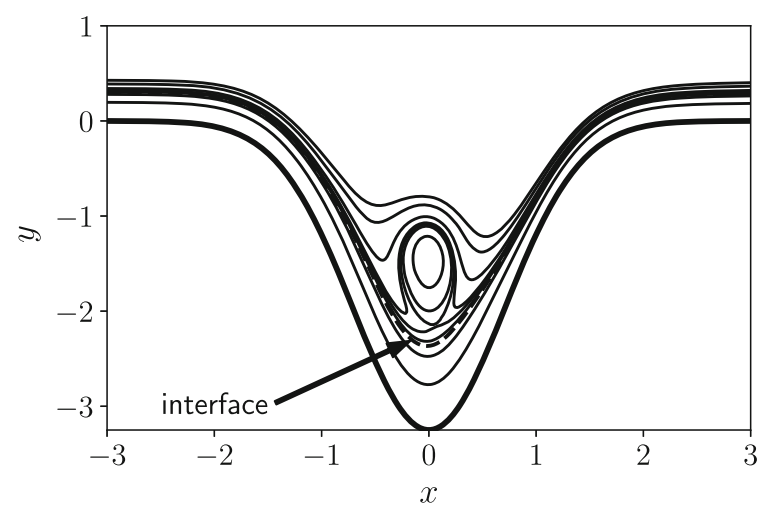

(d) $a=0.3$

Fig. 6 Varying film thickness with $K>0$ leads to a pair of vortices joined at the interface initially transforming into a single vortex in the upper fluid. b Streamlines near the first flow reversal in the upper fluid in a two-vortex configuration. The dashed curve is the interface

An even more complicated chain of transformations emerges if the incoming film is very thin initially and the channel slope increases. Illustrations in Fig. 7 are for the indentation of depth $h_{0}=-3$, the film fluid parameters are $\rho=1.5, \mu=2.25$, the incoming film thickness is $a=0.02$.

The non-sloping channel shows the standard on-wall separation reduced somewhat in size by a mild channel inclination, Fig. 7a, b. A further tilting of the channel gives rise to a new vortex on the interface, still preserving the remnants of the on-wall separation, Fig. 7c, d. An even bigger channel slope tends to eliminate flow separation in the film, first removing the on-wall vortex and then pushing the interfacial vortex into the upper fluid, Fig. 7e, f.

\subsection{Transverse gravitational force: upstream influence}

In all previous computations, the effect of the interfacial pressure jump due to the transverse pressure variation was neglected by assuming $g=0$ in (37). The main new physical effect associated with non-zero transverse gravity is upstream propagation of disturbances. If, for instance, a fluid particle in the near-wall fluid experiences a sudden deceleration then the interface is displaced upwards leading to a pressure rise at the wall and hence a further deceleration in the flow allowing a self-sustained feedback. For a single liquid layer bounded by a free surface, the transverse gravitational pressure supports upstream propagation of disturbances as an essential element of a hydrolic jump [27,28]. A related effect in a two-fluid flow is studied in [29] in the context of aircraft icing research. The 


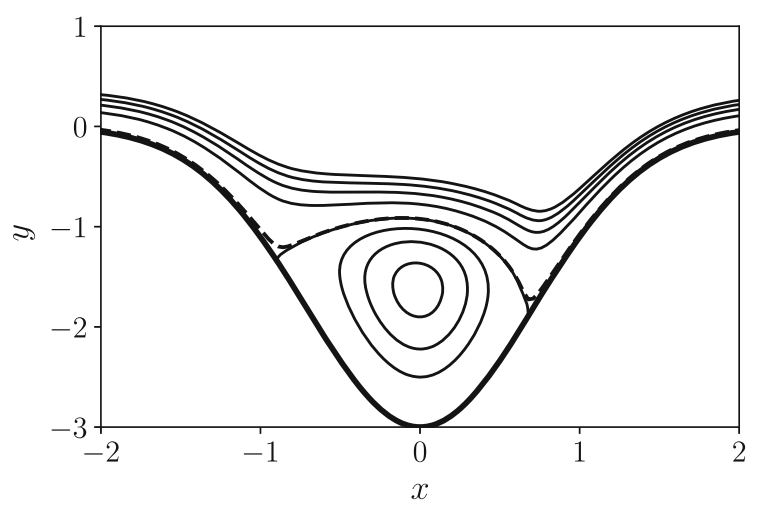

(a) $K=0$, no slope

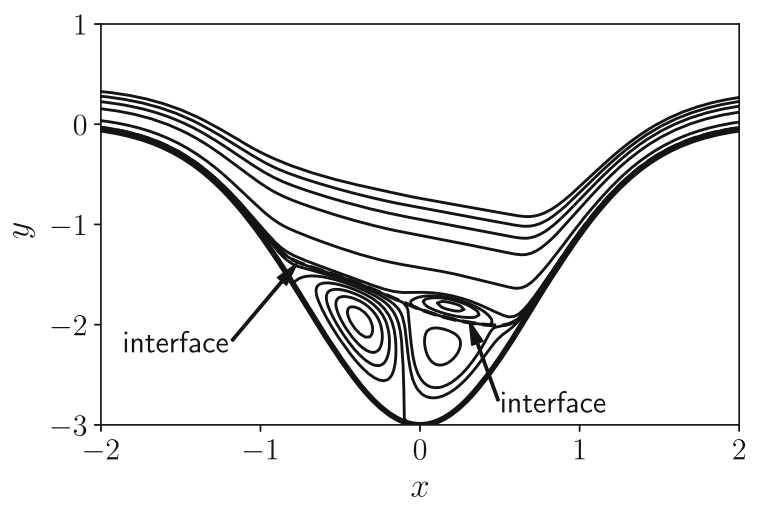

(c) $K=0.3$

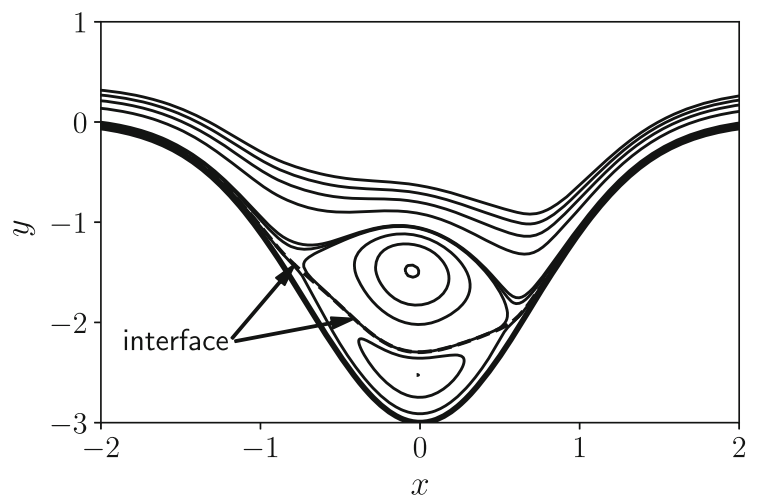

(e) $K=0.5$

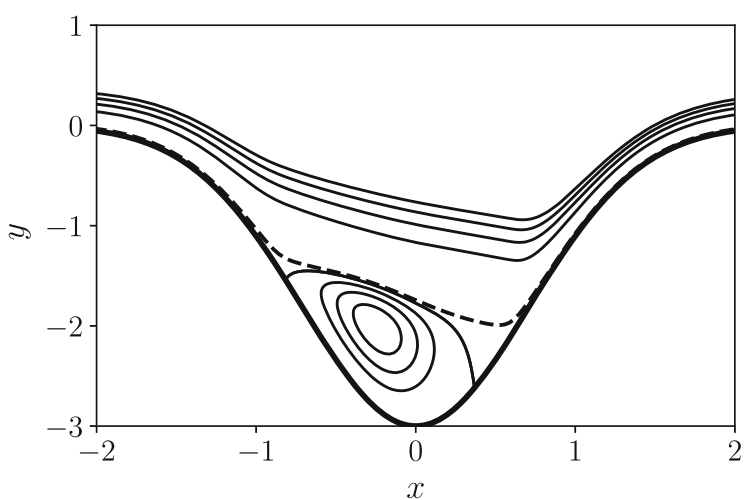

(b) $K=0.27$

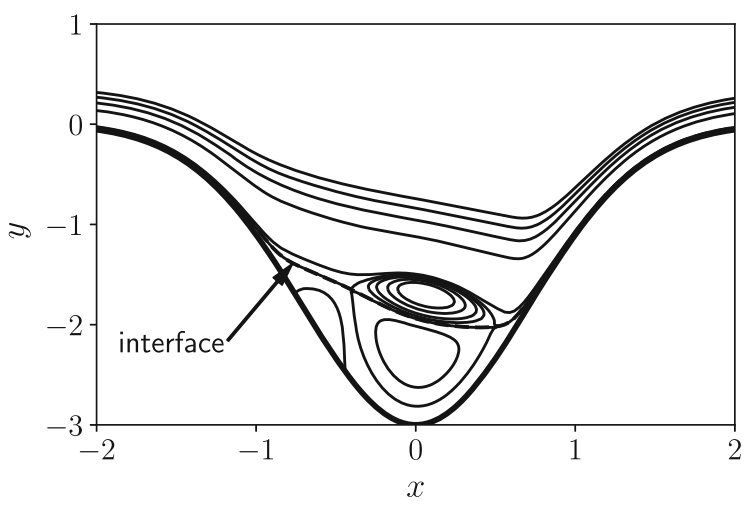

(d) $K=0.33$

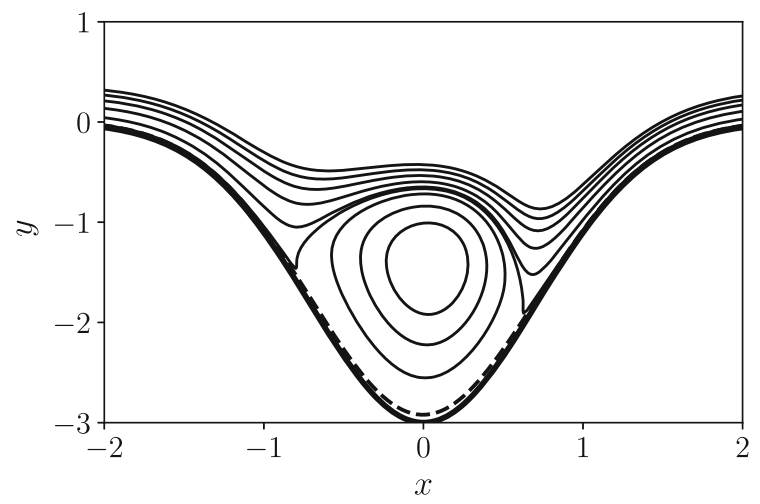

(f) $K=5$

Fig. 7 Increasing the channel slope leads to a transformation of an on-wall into an interior separation with the interfacial vortex appearing in the intermediate stages. The dashes show the interface

flow regime in [29] corresponds to a condensed viscous layer adapted to the air/water combination with the large density and viscosity ratios between fluids captured in what is termed weak or strong lubrication approximation, depending on the flow specifics. Upstream influence in that study is believed to be featuring in the dam effect noted ahead of surface roughness.

In our case, the coupling between the two fluids at the interface is stronger than that in [29] and any transverse displacements of the interface need to be balanced with the requirement of a vanishing displacement in the two 


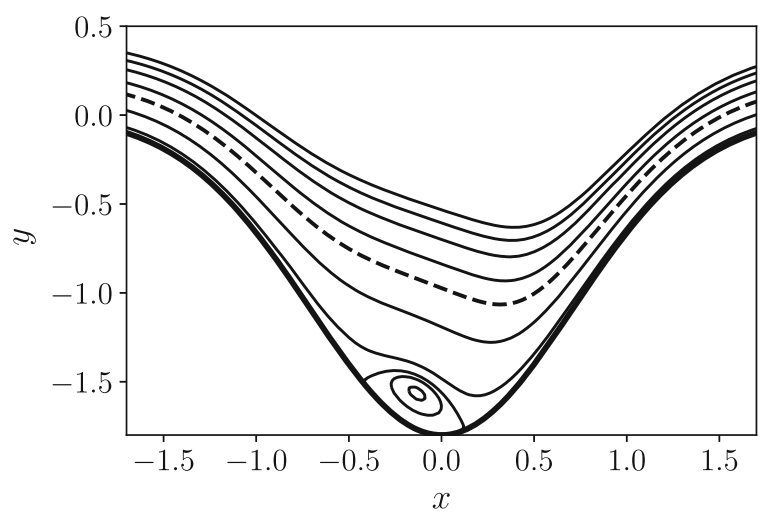

(a) Subcritical flow, $g=0$

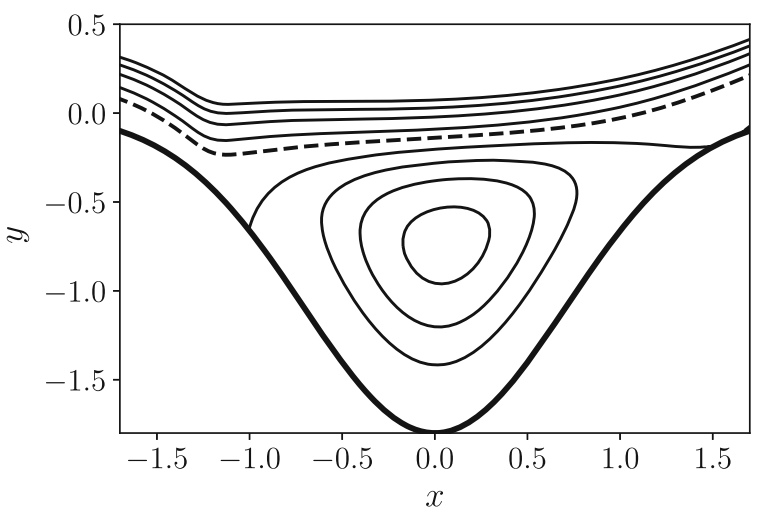

(b) Supercritical flow, $g=10$

Fig. 8 The effect of transverse gravity on the flow past a dent. In both cases, $K=0$

fluids combined. A straightforward calculation based on a locally inviscid approximation shows that the flow allows upstream influence if $g>g_{c}=a / \mu$ provided that the channel remains strictly horizontal. Acknowledging some differences in historic terminology, we refer to the flow with $g<g_{c}$ as subcritical (supercritical otherwise). A detailed description of sub/spercritical boundary layers is beyond the scope of this paper but in connection with the material above, we will show one example of a computed flow field past an indentation in a horizontal channel $(K=0)$ influenced by a rather strong transverse gravitational pressure.

The flow in Fig. 8 has $\rho=2, \mu=4$ and the incoming film thickness $a=0.2$. The depth $h_{0}=-1.8$ corresponds to an incipient on-wall flow reversal in the subcritical flow without gravity, Fig. 8a. Gravity in the transverse direction in Fig. $8 \mathrm{~b}$ is seen to promote a stronger flow reversal due to a flattening of the interface and an additional expansion of the fluid filaments in the near-wall region.

\section{Discussion}

The examples in this paper serve to show that several topologies of a reversed-flow field are possible in a twofluid boundary layer, some highly fragmented, even in a simple geometry. The appearance of a reversed doublevortex at the interface with a parametric evolution into a single vortex displaced into the upper or lower fluid is especially interesting. Similar trends can be expected in a time-dependent flow, with obvious implications for the issue of mixing. Although a technical extension to unsteady flows is straightforward, the physics becomes more complicated. First of all, the flow becomes susceptible to various instabilities as in [15]. In pre-separated flows, inflexional instabilities are a norm [17], and the whole issue is exacerbated further by a potential absolute instability of any extended reversed-flow regions [30]. The streamline patterns in Figs. 6, 7 in this paper suggest fairly complicated stability properties in two-fluid separated flows.

One conclusion to draw from the reported computations is that the first point of flow reversal in an otherwise forward current does not necessarily occur on a closed streamline, see Fig. $6 \mathrm{~b}$. This correlates with observations made in trailing-edge flows in [31] and suggests that, in general, an interior flow reversal may occur without the formation of a closed vortex. The local modelling in Sect. 4.5 certainly allows it as a theoretical possibility.

An important extension of this work will follow with the inclusion of interfacial surface tension, other types of viscous/inviscid interaction and, of course, with a deeper study of upstream influence and possible trans-critical regimes in the case of non-negligible transverse gravitational forces. With regard to the latter, Fig. 8 gives just a hint of what may happen in a flow field dominated by gravitational forces, with a further clear prospect of a self-induced separation or other related processes observed, for example, in a single liquid layer, see e.g. [27,28,32], and in a two-fluid flow in [29]. Preliminary computations based on the codes used for this paper give encouraging results but the numerical method proves inefficient wherever large-scale global iterations are needed. 
Another interesting lead from the results in this paper is contained in Fig. 2. We refer to the fact that the reversed bubble in an on-wall separation appears to be completely filled with the film fluid even for an infinitesimally thin incoming film. It is tempting to suggest that a flow with a separation region filled with a different fluid may be possible if the incoming film flow is 'switched off', in some analogy with the idea of a pre-cursor film used in modelling of spreading liquid drops, e.g. [33].

A further interesting option to consider, still within the channel setting, is a base flow dominated by the Poiseuille component with the velocity of the moving wall acting as a free additional parameter. When the wall velocity is sufficiently small, a viscous/inviscid interaction arises on a long length scale due to pressure variations across the channel, with the displacement of the viscous sublayer no longer negligible as in [10]. Related structures for a single fluid were examined in the context of flow stability in [34]. Counter-flowing films as, for instance, in an upward sloping channel, is yet another possibility, adding to a vast range of aspects not nearly enough addressed in this paper.

Open Access This article is distributed under the terms of the Creative Commons Attribution 4.0 International License (http:// creativecommons.org/licenses/by/4.0/), which permits unrestricted use, distribution, and reproduction in any medium, provided you give appropriate credit to the original author(s) and the source, provide a link to the Creative Commons license, and indicate if changes were made.

\section{Appendix A: Parameters in the Poiseuille-Couette velocity profile}

The constants $D^{ \pm}$in (7), (8) can be represented as follows. Let

$r_{1}=\frac{\mu^{+} \pi^{+}-\mu^{-} \pi^{-}}{\mu^{+}}, \quad r_{2}=\frac{\pi^{+}}{2}\left(y_{i}^{2}-1\right)-\frac{\pi^{-}}{2} y_{i}^{2}-1$,

then

$D^{-}=\mu^{+} \frac{r_{2}-r_{1}\left(y_{i}-1\right)}{\mu^{-}\left(y_{i}-1\right)-\mu^{+} y_{i}}, \quad D^{+}=\frac{\mu^{-}}{\mu^{+}} D^{-}+r_{1}$.

In the limit of a thin layer, we have $y_{i}=\varepsilon a_{0}, \pi^{-}=\varepsilon^{-1} \bar{\pi}^{-}+\cdots$, hence

$$
\begin{aligned}
& r_{1}=-\frac{\mu^{-}}{\mu^{+}} \bar{\pi}^{-}+O(\varepsilon), \\
& r_{2}=-\left(\frac{\pi^{+}}{2}+1\right)-\frac{\bar{\pi}^{-}}{2} a_{0}^{2} \varepsilon+O\left(\varepsilon^{2}\right),
\end{aligned}
$$

leading to the expansions

$$
\begin{aligned}
& D^{+}=\frac{\pi^{+}}{2}+1+\varepsilon\left[a_{0}\left(\frac{\pi^{+}}{2}+1\right)\left(1-\frac{\mu^{-}}{\mu^{+}}\right)-\frac{\bar{\pi}^{-} a_{0}^{2}}{2}\right]+O\left(\varepsilon^{2}\right), \\
& D^{-}=\frac{\mu^{+}}{\mu^{-}}\left(\frac{\pi^{+}}{2}+1\right)+\bar{\pi}^{-} a_{0}+O(\varepsilon) .
\end{aligned}
$$

The expansions for the base-flow velocity profiles can now be derived from (7), (8).

\section{Appendix B: The core and viscous sublayer on the upper channel wall}

Here we show that the viscous sublayer on the lower wall has no displacement effect at the leading order as stated in (28) and derive leading-order terms in the expansions of the flow functions outside the viscous sublayer on the lower 
wall. In the core of the channel, where $y=O(1)$, matching with (27) assuming a non-negligible displacement for now, we have

$$
\begin{aligned}
u^{+} & =U_{0}^{+}(y)+\varepsilon\left[U_{0}^{+}(Y)+\widehat{U}_{1}(X, y)\right]+\varepsilon^{2}\left[U_{2}^{+}(Y)+\widehat{U}_{2}(X, y)\right]+\cdots, \\
v^{+} & =\frac{\varepsilon}{L} \widehat{V}_{1}(X, y)+\frac{\varepsilon^{2}}{L} \widehat{V}_{2}(X, y)+\cdots, \\
p^{+} & =P^{+}+\varepsilon^{2} \widehat{P}_{2}(X, y)+\cdots,
\end{aligned}
$$

where the terms $U_{0}^{+}, U_{1}^{+}, \cdots$ are the base flow components as in (10), $P^{+}$denotes the base-flow pressure and the hat designates contributions due to the wall obstacle. We take $\rho^{+}=\mu^{+}=1$ due to non-dimensionalisation and then, on substitution into (1)-(3), at the leading order, we obtain

$\widehat{U}_{1}=A_{1}(X)\left[U_{0}^{+}(y)\right]^{\prime}, \quad \widehat{V}_{1}=-A_{1}^{\prime}(X) U_{0}^{+}(y)$.

This suggests a non-vanishing transverse velocity component at the upper channel wall at $y=1$ where $U_{0}^{+}(1)=1$, therefore $A_{1}(x) \equiv 0$ taking into account that far upstream the obstacle-induced disturbances decay. A similar vanishing displacement effect is observed in certain regimes of the viscous-inviscid interaction in a Poiseuille channel flow, see [8-10]. It also follows that $\widehat{P}_{2}=\widehat{P}_{2}(x)$ with the pressure variation across the channel due to centrifugal forces (i.e. the term $u v_{x}$ in (2)) appearing at $O\left(\varepsilon^{2} / L^{2}\right)$ in the pressure expansion (43). At the next order in (41), (42), it is found that

$\widehat{U}_{2}=-\widehat{P}_{2}(X) \frac{\mathrm{d}}{\mathrm{d} y}\left\{U_{0}^{+} \int_{1}^{y} \frac{\mathrm{d} s}{\left[U_{0}^{+}(s)\right]^{2}}\right\}, \quad \widehat{V}_{2}=\frac{\mathrm{d} \widehat{P}_{2}}{\mathrm{~d} X} U_{0}^{+} \int_{1}^{y} \frac{\mathrm{d} s}{\left[U_{0}^{+}(s)\right]^{2}}$,

again using the no-penetration condition, $\widehat{V}_{2}(y=1)=0$.

In the viscous sublayer arising near the upper channel wall, let $y=1+\varepsilon^{3 / 2} z$ with $z=O(1)$, with the leadingorder expansions of the form:

$u^{+}=1+\varepsilon^{2} \tilde{u}(X, z)+\cdots, \quad v^{+}=\frac{\varepsilon^{7 / 2}}{L} \tilde{v}(X, z)+\cdots, \quad p^{+}=P^{+}+\varepsilon^{2} \tilde{p}(X, z)+\cdots$.

It follows that $\tilde{p}=\widehat{P}_{2}(X)$, that is no transverse pressure variation is found at this order either, with the solution for the velocity components derived via Fourier transforms, for example, in terms of the streamfunction $\tilde{\psi}$ such that $\tilde{u}=\partial \tilde{\psi} / \partial z$ and

$$
\tilde{\psi}=-\widehat{P}_{2} z+\frac{1}{2 \pi} \int_{-\infty}^{\infty}(\mathrm{i} k)^{-1 / 2} e^{i k X}\left[\mathrm{e}^{(\mathrm{i} k)^{1 / 2} z}-1\right] \widehat{P}_{2}(k) \mathrm{d} k,
$$

where the Fourier transform of the pressure is defined by

$$
\widehat{P}_{2}=\int_{-\infty}^{\infty} \mathrm{e}^{-\mathrm{i} k X} \widehat{P}_{2}(X) \mathrm{d} X
$$

As $z \rightarrow-\infty$, i.e. on approach to the core of the flow, we have $\tilde{\psi}=-\widehat{P}_{2} z+O(1)$ therefore $\tilde{v}(X, z)=$ $-\partial \tilde{\psi} / \partial X=z \mathrm{~d} \widehat{P}_{2}(X) / \mathrm{d} x+O(1)$. The last relation shows that, in terms of the transverse velocity, the displacement effect of the viscous sublayer near the upper wall is of order $\varepsilon^{7 / 2} / L$ and therefore does not feature in the expansions (41)-(43).

\section{Appendix C: Numerical method}

Numerical method is based on a mixed vorticity/velocity formulation. In the upper fluid, Prandtl's transposition is used to reduce the solution domain to a half-plane. Taking $y=Y+f(x), v^{+}=V^{+}+u^{+} f^{\prime}(x)$ and introducing 
the vorticity $\omega^{+}=\partial u^{+} / \partial Y$, the system of equations (30) is written as

$u^{+} \omega_{x}^{+}+V^{+} \omega_{Y}^{+}=\omega_{Y Y}^{+}, \quad u_{x}^{+}+V_{Y}^{+}=0, \quad u^{+}=\int_{0}^{Y} \omega^{+}(x, s) \mathrm{d} s+u_{s l}(x)$,

where the flow functions are defined in $Y \geq 0,-\infty<x<\infty ; u_{s l}(x)$ is the interfacial slip velocity, and the following boundary conditions hold,

$$
\begin{aligned}
& \left.\omega_{Y}^{+}\right|_{Y=0}=\left.u^{+} u_{x}^{+}\right|_{Y=0}+\left[p^{+}(x)\right]^{\prime}, \\
& \left.V^{+}\right|_{Y=0}=0,\left.\quad \omega^{+}\right|_{Y \rightarrow \infty}=1,\left.u^{+}\right|_{Y \rightarrow \infty}=Y-a+u_{S}+f(x)+o(1) .
\end{aligned}
$$

In the lower fluid, Prandtl's transposition is combined with mapping the flow region onto a unit strip. The idea of this transformation was suggested to us by Dr J.W. Elliott around 1999 in private communication. Using the film thickness $H(x)=f(x)-h(x)$, the new transverse coordinate $z$ is defined by $y=h(x)+H(x) z$, with the vorticity $\omega^{-}=\partial u^{-} / \partial y$. The transverse velocity component is modified,

$\tilde{v}^{-}=v^{-}-u^{-} h^{\prime}(x)-z H^{\prime}(x) u^{-}$,

leading to the equations of motion of the form

$u^{-} \omega_{x}^{-}+\frac{\tilde{v}^{-}}{H} \omega_{z}^{-}=\frac{\mu}{\rho H^{2}} \omega_{z z}^{-}, \quad\left(H u^{-}\right)_{x}+\tilde{v}_{z}^{-}=0, \quad u^{-}=H(x) \int_{1}^{z} \omega^{-}(x, s) \mathrm{d} s+u_{s l}(x)$.

The solution domain is $0 \leq z \leq 1,-\infty<x<\infty$. The boundary conditions are

$u^{-}=\tilde{v}^{-}=0 \quad$ at $z=0,\left.\quad \tilde{v}^{-}\right|_{z=1}=0,\left.\quad \rho\left(u^{-} u_{x}^{-}\right)\right|_{z=1}=-\left(p^{-}\right)^{\prime}+K+\left.\frac{\mu}{H} \omega_{z}^{-}\right|_{z=1}$.

Since $u^{+}=u^{-}=u_{s l}$ at the interface, the vorticity gradients in (44), (45) can be combined into a single jump requirement which, together with continuity of the interfacial tangential stress, gives two convenient boundary conditions,

$\left.\omega_{Y}^{+}\right|_{Y=0}-\left.\frac{\mu}{H} \omega_{z}^{-}\right|_{z=1}=(1-\rho) u_{s l} u_{s l}^{\prime}+\left(p^{+}-p^{-}\right)^{\prime}+K,\left.\quad \omega^{+}\right|_{Y=0}-\left.\mu \omega^{-}\right|_{z=1}=0$.

A second-order accurate marching procedure is used with central differences to approximate transverse derivatives and three-point backward approximations for the $x$-derivatives. Starting from the specified undisturbed flow upstream of the obstacle, guesses are made on the transverse vorticity at a new $x$-station from which the velocity components are restored. A new vorticity field is computed via a Thomas algorithm and the interfacial conditions are then used to update the interfacial slip velocity and the value of the pressure jump. The solution is then iterated essentially in two loops: to accommodate the nonlinearity in the momentum equations and to satisfy the interfacial conditions, the latter achieved by means of Newton's iterations. When a region of reversed flow is encountered, three approaches have been tried: continuing marching in the positive $x$-direction (this only works on coarse grids and for very small regions of reversed flow), FLARE approximation [35], and switching to a forward approximation for the $x$-derivatives at the points with negative flow velocity hence employing global iterations. The FLARE method and global iterations give graphically indistinguishable results for the flows computed in this paper, in accord with the conclusions drawn in [36]. The grids employed had typically $x \in[-3,3], y \in[0,6]$ with 200 or 400 grid points in each direction in the upper fluid, and usually 50-100 grid points in the film, depending on the incoming film thickness. As part of the validation exercise, the two-fluid code implemented in Python 2.7 was run for the case of two identical fluids and compared with a single-fluid Matlab code (with a different implementation of nonlinear iterations). The difference between two numerical solutions on grids from $100 \times 100$ up to $500 \times 500$ proved within the convergence tolerance for both non-separated and reversed-flow regimes. 


\section{References}

1. Ruban AI (2017) Fluid dynamics: part 3 boundary layers. Oxford University Press, Oxford

2. Smith FT (1982) On the high Reynolds number theory of laminar flows. IMA J Appl Math 28(3):207-281

3. Smith FT (2012) On internal fluid dynamics. Bull Math Sci 2(1):125-180

4. Sobey IJ (2000) Introduction to interactive boundary layer theory, vol 3. Oxford University Press, Oxford

5. Timoshin SN (2016) Multiple deck theory. In: Bullett S (ed) Fluid and solid mechanics. World Scientific, London, pp 35-69

6. Szeri AZ (2010) Composite-film hydrodynamic bearings. Int J Eng Sci 48(11):1622-1632

7. Severtson YC, Aidun CK (1996) Stability of two-layer stratified flow in inclined channels: applications to air entrainment in coating systems. J Fluid Mech 312:173-200

8. Smith FT (1976) Flow through constricted or dilated pipes and channels: part 1. Quart J Mech Appl Math 29(3):343-364

9. Smith FT (1976) Flow through constricted or dilated pipes and channels: part 2. Quart J Mech Appl Math 29(3):365-376

10. Smith FT (1977) Upstream interactions in channel flows. J Fluid Mech 79(4):631-655

11. Hunt JCR (1971) A theory for the laminar wake of a two-dimensional body in a boundary layer. J Fluid Mech 49(1):159-178

12. Bogolepov VV, Neiland VY (1971) Viscous gas motion near small irregularities on a rigid body surface in supersonic flow. Trans TsAGI 1363

13. Smith FT, Brighton PWM, Jackson PS, Hunt JCR (1981) On boundary-layer flow past two-dimensional obstacles. J Fluid Mech 113:123-152

14. Lynn J, Rothmayer AP (1993) Viscous flows past short-scaled humps and surface blowing. Eur J Mech B 12(3):313-321

15. Timoshin SN (1997) Instabilities in a high-Reynolds-number boundary layer on a film-coated surface. Journal of Fluid Mechanics 353:163-195

16. Pelekasis NA, Tsamopoulos JA (2001) Linear stability of a gas boundary layer flowing past a thin liquid film over a flat plate. J Fluid Mech 436:321-352

17. Caporn PA (1998) Linear and nonlinear instability of shear-driven liquid films. PhD thesis, University College London (University of London)

18. Goldstein S (1948) On laminar boundary-layer flow near a position of separation. Quart J Mech Appl Math 1(1):43-69

19. Rothmayer AP, Matheis BD, Timoshin SN (2002) Thin liquid films flowing over external aerodynamic surfaces. J Eng Math 42(3-4):341-357

20. Schweizer PM (1988) Visualization of coating flows. J Fluid Mech 193:285-302

21. Vlachomitrou M, Pelekasis N (2009) Nonlinear interaction between a boundary layer and a liquid film. J Fluid Mech 638:199-242

22. Cimpeanu R, Papageorgiou D, Kravtsova M, Ruban A (2015) The effect of thin liquid films on boundary-layer separation. In: APS meeting abstracts

23. Luo H, Blyth MG, Pozrikidis C (2008) Two-layer flow in a corrugated channel. J Eng Math 60(2):127-147

24. Timoshin SN (1996) Concerning marginal singularities in the boundary-layer flow on a downstream-moving surface. J Fluid Mech 308:171-194

25. Heil M, Rosso J, Hazel AL, Brøns M (2017) Topological fluid mechanics of the formation of the Kármán-vortex street. J Fluid Mech 812:199-221

26. Dam M, Juul Rasmussen J, Naulin V, Brøns M (2017) Topological bifurcations in the evolution of coherent structures in a convection model. Phys Plasmas 24(8):082301

27. Bowles RI (1990) Applications of nonlinear viscous-inviscid interactions in liquid layer flows and transonic boundary layer transition. PhD thesis, University College London (University of London)

28. Bowles RI (1995) Upstream influence and the form of standing hydraulic jumps in liquid-layer flows on favourable slopes. J Fluid Mech 284:63-96

29. Rothmayer AP, Zhang K, Hu H (2016) Gravitational effects in low-speed air-driven films. In: 8th AIAA atmospheric and space environments conference, $\mathrm{p} 3141$

30. Logue RP, Gajjar JSB, Ruban AI (2011) Global stability of separated flows: subsonic flow past corners. Theor Comput Fluid Dyn 25(1-4):119-128

31. Smith FT (1983) Interacting flow theory and trailing edge separation-no stall. J Fluid Mech 131:219-249

32. Scheichl B, Bowles RI, Pasias G (2018) Developed liquid film passing a trailing edge under the action of gravity and capillarity. J Fluid Mech 850:924-953

33. Diez JA, Kondic L, Bertozzi A (2000) Global models for moving contact lines. Phys Rev E 63(1):011208

34. Cowley SJ, Smith FT (1985) On the stability of Poiseuille-Couette flow: a bifurcation from infinity. J Fluid Mech 156:83-100

35. Reyhner TA, Flügge-Lotz I (1968) The interaction of a shock wave with a laminar boundary layer. Int J Non-Linear Mech 3(2):173199

36. Chouly F, Lagrée PY (2012) Comparison of computations of asymptotic flow models in a constricted channel. Appl Math Model 36(12):6061-6071

Publisher's Note Springer Nature remains neutral with regard to jurisdictional claims in published maps and institutional affiliations. 